\title{
A computational model-based approach for atlas construction of aortic Doppler velocity profiles for segmentation purposes
}

\author{
Vedrana Baličevića ${ }^{\text {, Hrvoje Kalinić }}{ }^{\text {, Sven Lončarića }}{ }^{\text {, }}$ \\ Maja Čikešc ${ }^{c}$, Bart Bijnens, ${ }^{\mathrm{d}, e, f}$ \\ ${ }^{a}$ University of Zagreb Faculty of Electrical Engineering and Computing, Croatia \\ ${ }^{b}$ University of Split, Faculty of Science, Croatia \\ ${ }^{c}$ University of Zagreb School of Medicine, Department of Cardiovascular Diseases, Croatia \\ ${ }^{d}$ PhySense, Universitat Pompeu Fabra, Barcelona, Spain \\ 'ICREA, Barcelona, Spain \\ ${ }^{f}$ KU Leuven, Belgium
}

Email address: vedrana.balicevic@fer.hr (Vedrana Baličević) 


\begin{abstract}
Echocardiography is the leading imaging modality for cardiac disorders in clinical practice. During an echocardiographic exam, geometry and blood flow are quantified in order to assess cardiac function. In clinical practice, these imagebased measurements are currently performed manually. An automated approach is needed if more advanced analysis is desired.

In this article, we propose a new hybrid framework for the construction of a disease-specific atlas to improve Doppler aortic outflow velocity profile segmentation. The proposed method is based on combining realistic computational simulations of the cardiovascular system for common cardiac conditions (using CircAdapt) with a validated image-based atlas construction method. The coupling is realized via model-based generation of echocardiographic images of virtual populations with a statistically approved parameter variation. We created virtual populations of 100 healthy individuals and 100 patients with aortic stenosis, synthesized their aortic Doppler velocity images and constructed the corresponding atlases. We validated atlases' performances by comparing their segmentation of real clinical images with the manually segmented ground truth. The experimental results show that the segmentation accuracy obtained using the proposed atlases is comparable to the accuracy obtained using classical clinical image-based atlases. Moreover, this framework eliminates the time-consuming acquisition of a sufficient number of representative images in clinical practice, offering a substantial time efficiency and flexibility in creating a disease specific atlas and ensuring an observer-independent automated segmentation. The proposed approach can easily be extended towards the creation of atlases for segmenting any Doppler trace in the cardiovascular circulation in a specific disease.
\end{abstract}

Keywords: Echocardiography, velocity profile, aortic valve, CircAdapt, virtual population, atlas, image segmentation, image registration 


\section{Introduction}

Clinical imaging of cardiovascular structure and function often results in images that need further analysis for an accurate diagnosis. This analysis can depend on the underlying disease process. For example, in aortic stenosis, the orifice of the aortic valve is narrowed, causing partial obstruction of the blood flow from the heart into the aorta and onward to the rest of the body. If left untreated, it can lead to heart failure. Doppler echocardiography of the blood flow through the valve is the standard procedure for evaluating suspected valvular heart disease in a patient. Specifically, in continuous-wave (CW) Doppler mode, all velocity values along a scanline are detected simultaneously, forming a velocity spectrum in time. Since the fastest blood flow is expected within the valve, diagnostic information is encoded in the spectral envelope. Diagnosis is based on valuation of the maximum blood flow velocities (amplitude of the envelope) and the transaortic pressure gradient (area under the curve). (Baumgartner et al. (2009)). Additional computational analysis of transaortic outflow profile attributes may infer latent, but discriminative information about the valvular condition and its influence on the left ventricular function (Čikeš et al. (2009)). The extraction of the spectral trace envelope for a detailed analysis represents an image segmentation problem.

While manual evaluation of the spectral amplitude is common in clinical practice, it is characterized by high inter- and intra-observer variability of both the velocity value (Otto (2013)) and the severity of heart valve disease (Kupfahl et al. (2004)). A precise manual delineation would enable a more detailed analysis of the aortic outflow during specific phases of a cardiac cycle. However, it is time-consuming and thus only performed roughly in order to improve patient throughput in echocardiographic laboratories. Automated segmentation is a solution that can overcome these disadvantages - it is faster, accurate, consistent and reproducible, thus facilitating and supporting clinical image interpretation and immediate patient evaluation. Also, it allows for a computational analysis of the blood flow profile features, which is especially useful for resolving possible ambiguities in diagnosing conditions with similar clinical pictures.

Contemporary image segmentation approaches are often based on the use of representative atlases created from real-world images and their labellings (Kalinić (2008)). However, for segmentation of images with disease-specific alterations captured at hand, their performance is highly dependent on the quantity and the representativeness of the acquired images and the preciseness of the performed labellings. In other words, it relies on availability and variability of patients and

time costly manual delineation of all acquired images. To overcome this, we 
offer an alternative where the images can be computationally simulated for the condition of interest.

In this article, we describe the novel approach towards atlas construction for automated atlas-based segmentation of aortic outflow Doppler profiles, in order to support evidence-based diagnosis of cardiac diseases with high accuracy. Instead of working with acquired patient images, we propose to create the data set independently by using a computational model capturing the relevant pathophysiological changes. In this study we use the CircAdapt model (Arts et al. (2005); Lumens et al. (2009)), validated for different cardiovascular conditions, to simulate rich populations of healthy individuals and patients with (different degrees of) aortic stenosis. Inter-individual diversity is achieved by varying the model parameters over an expected range of values for each population. The model simulation results can be transformed into virtual echocardiographic images using a modality-specific imaging simulator (Palau-Caballero et al. (2013)), which further serve as input for anatomical atlas construction. The more specific an atlas is to the structure that is being segmented, the registration algorithm will converge faster to a more accurate solution. Even though a Doppler valve velocity profile appears to be a simple structure, for different valves, different valve conditions and different conditions severities, these profiles can have quite different shapes. This is the reason for introducing disease-specific atlases into the process of automatic image segmentation. This coupled computational modelling and velocity profile atlas construction is validated for atlas-based segmentation in a specific clinical population. The pipeline of the coupling is visualized in Fig.1, together with all steps conducted in the experiment.

Since the proposed method unites different research areas, a brief overview of each of them is given below.

\section{Atlas-based segmentation of medical images}

In the past five decades, the automatic analysis of anatomical structures in medical images has become a valuable tool for clinical practice. Amongst a variety of existing approaches (Pham et al. (2000); Ma et al. (2010)), atlas-based segmentation has proven to be an efficient method, with the benefit of introducing a priori knowledge into the process of segmentation. An anatomical atlas provides the "default" anatomical structure within the observed population, with a label for each of the structures. By propagating its segmentation and labels to a new image, the same structures in the new image can be identified.

The majority of atlases for segmentation have been derived from a set of representative clinical images. Simple techniques for atlas construction employ random 


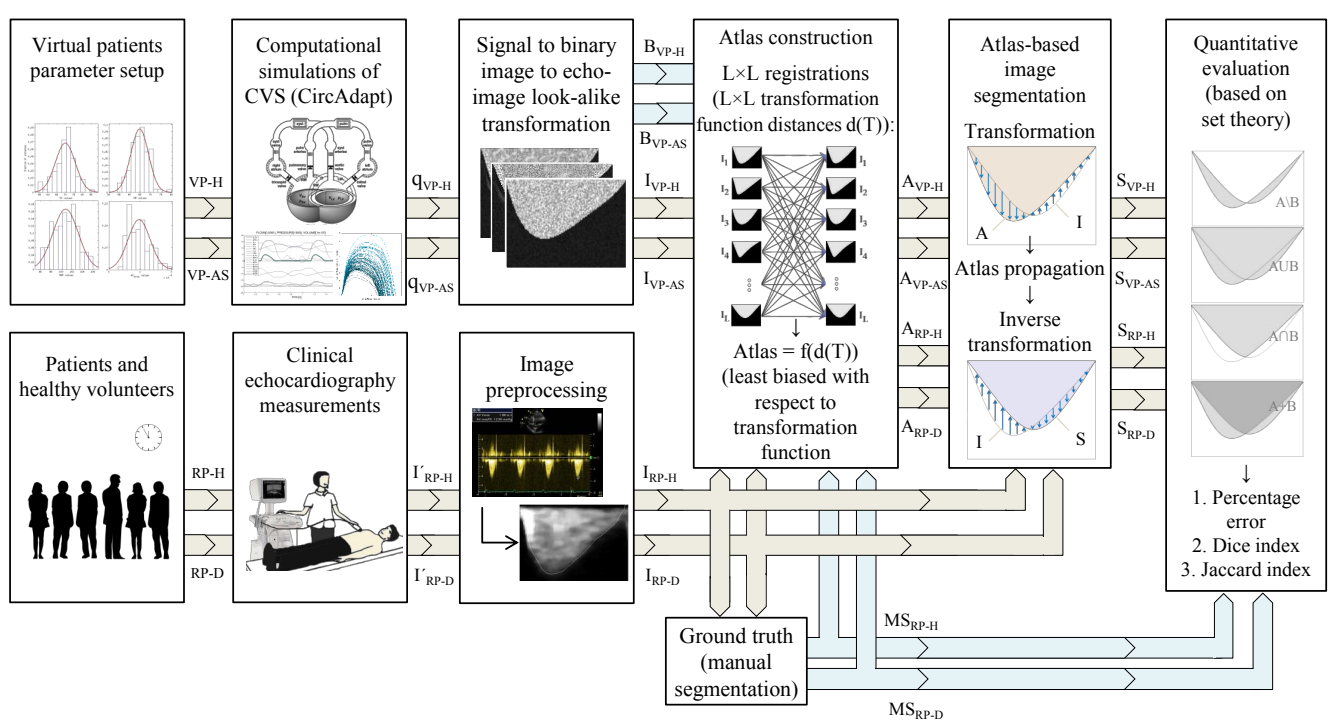

Legend: $\mathrm{VP}=$ Virtual population, $\mathrm{RP}=$ Real population, $\mathrm{H}=$ healthy, $\mathrm{D}=$ diseased, $\mathrm{AS}=$ Aortic stenosis, $\mathrm{q}=$ flow, $\mathrm{I}^{\prime}=$ raw image, $\mathrm{I}=$ preprocessed image, $\mathrm{B}=$ binary image, $\mathrm{A}=$ atlas, $\mathrm{S}=$ image segmented using an atlas, $\mathrm{MS}=$ manually segmented image.

Figure 1: Flowchart of the performed experiment. Following the upper branch of the flowchart corresponds to the proposed method of the aortic Doppler segmentation using an computational model-based atlas, whereas the rest of the steps represent the typical approach with a clinical-based atlas. The two methods are compared in the validation step.

selection within the image data set (Yishan and Chung (2011)) or taking the average intensity (mean or median), usually coupled with the average-shape (Gruslys et al. (2011); Madden (2007)). More complex methods utilize probabilistic calculus (Gubern-Mérida et al. (2011); Kuklisova-Murgasova et al. (2011)), or a multiatlas approach merging several atlas propagations into the resulting image (Wang et al. (2013)). The use of imaging modality simulators has been proposed to create large sets of images that can capture the appearance of clinical images for a particular modality, given knowledge on the content shape variation (Tobon-Gomez et al. $(2008,2012))$. In this paper, we extend this idea towards Doppler images and simulating both the content appearance in these images (using information on the imaging modality) as well as the content shape (using knowledge about physiological processes in cardiovascular system).

\section{Segmentation of aortic Doppler velocity profiles}

Even though quantification of blood flow velocity profiles is a frequent task in clinical practice, there has not been extensive research dealing with the problem of 
its automation. While the segmentation of velocity profiles reported by Bermejo et al. (2000) is manual, they emphasize the need for automatic assessment for improved diagnosis or clinical research purposes. Automatic segmentations of similar Doppler traces in heart valves (other than aortic), based on a series of simple image processing operations, were described by Tschirren et al. (2001) and Greenspan et al. (2005). Gaillard et al. (2010) describe the aortic and mitral Doppler wave segmentation using a more advanced active contour method, initialized with a shape of the centers of divergence of the gradient vector flow field, reporting sensitivity of the method to the image contrast. Motivated by the clinicians' need for not only fast and automatic delineation but also for automatic extraction of condition-descriptive mathematically-derived features from the aortic valve velocity profile shape, Kalinić et al. (2009a,b, 2012) applied and developed several methods for atlas-based segmentation of Doppler velocity images. Some of those are adapted and incorporated here as a part of our novel hybrid method.

\section{Computational modelling of the cardiovascular system}

The human cardiovascular system is a closed multiphysics system that can be described with mathematical equations. Several approaches have resulted in a large number of available lumped- and distributed-parameter computational models (NSR Physiome (2000); Shi et al. (2011)), and the two coupled (Liang et al. (2009)). Lumped-parameter or 0-D models simplify the anatomy by assuming a uniform distribution of fundamental variables within the compartments, but they also simplify the calculus and allow for larger (or even closed-loop) circulation systems to be modelled. Regardless of their simplification, these models are accurate enough to capture various properties of cardiovascular physiology. They have been used as an educational tool for understanding cardiovascular (patho)physiology and as a research tool in examining the effectiveness of therapy (pacemakers, valve replacements, pharmaceutical products etc.) (De Lazzari et al. (2000); Cavalcanti et al. (2004); Di Molfetta et al. (2010); Khalafvand et al. (2012); McCormick et al. (2014)). Coupling these models with clinical measurements represents the state-of-the-art in biomedical engineering, with a great perspective of integrating them into clinical practice.

Often, the parameters of the computational cardiac models (such as radiuses, lengths, walls thicknesses etc.), are set for an average healthy individual or even deduced from in-vitro experiments on different species. Since each person is characterized with their own set of parameter values (also depending on their health state), such generalization has inherent limitations and might not be representative for a population. 
The model we used in our study is a MATLAB implementation (Matlab (2015)) of CircAdapt, by T. Arts (Arts et al. (2005)) and J. Lumens (Lumens et al. (2009)). It is a lumped-parameter computer model that enables beat-to-beat simulation of the heart and circulatory dynamics. In most pathologies, a single physiological defect invokes a series of adaptive mechanisms in the rest of the system, in order to compensate for the reduced functionality of the defective part. In computer simulations, it means that the variation of a single or a small set of parameters should gradually (via an iterative adaptation algorithm) lead to a physiologically plausible and steady condition of interest. Following this idea, several pathologies were successfully simulated and studied previously with this model: pulmonary arterial hypertension (Lumens and Delhaas (2012)), left bundle branch block (Kuijpers et al. (2014), Leenders et al. (2012)), mitral valve regurgitation (Kuijpers et al. (2012)), and chronic thromboembolic pulmonary hypertension (Lumens et al. (2010)).

\section{Materials and methods}

The study complies with the Declaration of Helsinki and was approved by the University Hospital Wuerzburg review committee and the Ethics Committee of the Zagreb University Hospital Centre. Informed consent was obtained from all patients.

\subsection{Clinically obtained aortic outflow Doppler images}

In this study, we used images of the aortic outflow profiles recorded in CW Doppler mode with a state-of-the-art clinical echocardiographic scanner (Vivid 7, GE Healthcare). Using an apical 5-chamber view, the scanner records the realtime blood velocity through the aortic valve during several heart cycles and stores the data digitally in "raw" Dicom format. Further analysis of the images can be performed with an EchoPAC workstation (GE Healthcare) and relevant information can be exported for further analysis (Kalinić et al. (2012)).

The clinically acquired images were separated in two groups: healthy volunteers and a group of cardiac patients. The latter one covers two conditions that share some similar patho-physiological signs with regards to alterations of the aortic outflow velocity pattern: aortic stenosis (AS) and coronary artery disease (CAD). More precisely, the temporal evolution of the aortic Doppler flow in both

conditions shifts from early peaking during the ejection towards later peaking, so 
that the overall trace reshapes from triangular towards a much more rounded profile with a later maximal velocity. Considering this, both types of patients were acquired in order to capture the shape changes and increase the test set size.

A preprocessing step is done to separate and align heart cycles in all images. The zero-velocity line and maximal-velocity line were determined from a horizontal pixel projection, whereas the beginning and end of the ejection period were marked manually by an expert cardiologist. All images were cropped with respect to these four boundaries. For reduction of speckle noise, which characterizes the echocardiographic imaging, images were filtered with median filters $3 \times 3$ and $\lfloor h / 100\rfloor \times\lfloor w / 100\rfloor$, where $h$ and $w$ stand for height and width of the image. To complete virtual prealignment of images, all images were then scaled to dimensions of $100 \times 100$ pixels. Obtained images are used for validation of the proposed atlas approach and its comparison with a clinical-based atlas construction approach.

An example of the aortic outflow image that can be seen on the echocardiographic device during the examination is given in Fig.2(a). Examples of velocity spectra per ejection period, extracted from different echocardiographic sequences, are given in Fig.2 (b) and (c).

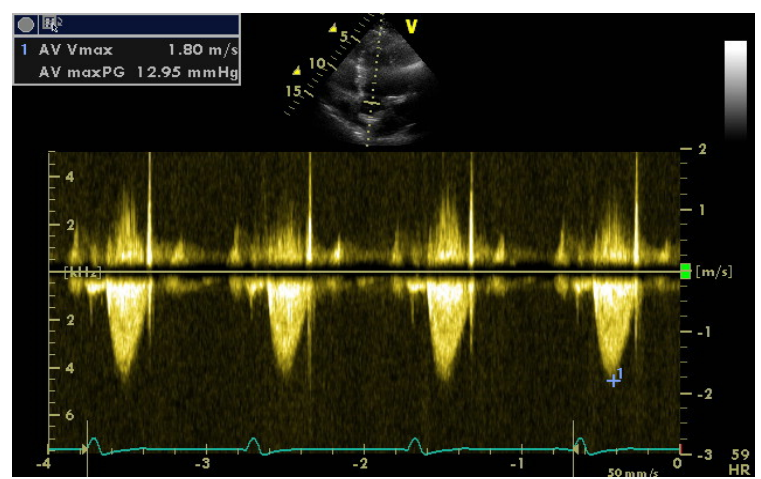

(a)

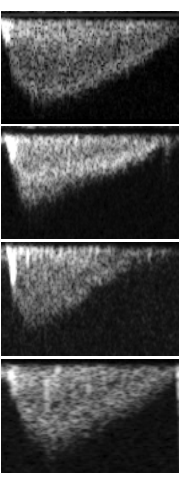

(b)

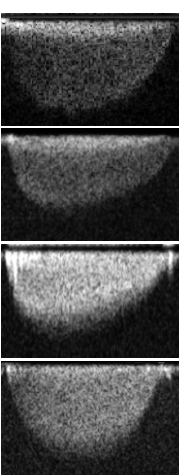

(c)

Figure 2: (a) An example of a CW Doppler recording of the aortic outflow shown on the echocardiographic scanner screen for a person with a healthy aortic valve. (b) Examples of cropped outflow images of healthy individuals. (c) Examples of cropped outflow images of patients with AS and CAD. Images were cropped with respect to the determined ejection periods.

\subsection{Virtual images obtained with CircAdapt}

CircAdapt model is based on a set of differential equations describing known physiological and hemodynamical principles in state-variables (flows, pressures, 
sarcomere lengths and contractilities) with respect to time. The equations are solved numerically with a MATLAB ODE solver.

The model was designed as a network assembled of four types of modules based on their anatomical equivalence to: cardiac chambers (left and right atria and ventricles), heart valves (mitral, aortic, tricuspid and pulmonary), large blood vessels (aortic and pulmonary arteries and pulmonary and systemic veins) and resistances (pulmonary and systemic peripheral vasculature). Its important advantage over other models is the incorporation of compensatory responses, which adapt the geometry and properties of the defined circulatory system to the induced mechanical load. Adaptation ensures that all cardiovascular system variables remain within biologically plausible ranges (Arts et al. (2005)).

A specific condition can be simulated with CircAdapt by setting the parameters of cardiac performance typical for that condition and iterating the simulations during adaptation until steady state is reached. This results in a single socalled virtual patient. To obtain a whole population of virtual patients, simulations should be repeated with a range of other typical values for the selected condition.

The virtual population of healthy individuals was simulated by varying the following parameters: stroke volume [mL/beat], heart rate [beats/min] and mean arterial pressure [mmHg]. The range of values for each of them was obtained by applying a normal distribution $G\left(\mu, \sigma^{2}\right)$, with mean value $\mu$ and standard deviation $\sigma$ chosen so that the parameters cover the values typical for a healthy population $^{1}$ (non-athletic and athletic individuals at rest) (Headley (2002); La Gerche et al. (2010)). All of the other model parameters were left unchanged.

The virtual population with aortic stenosis was derived from the healthy population (keeping their heart rate, stroke volume and mean arterial pressure values) by additionally changing the area of the aortic valve $\left[\mathrm{cm}^{2}\right]$. The aortic valve area was also described with a Gaussian distribution, covering values of the valve areas for mild, moderate and severe aortic stenosis. Other parameters that change with aortic stenosis (such as chamber sizes, walls thicknesses, ventricular pressures, etc.) were left to adapt for each individual by CircAdapt. The mean value and the standard deviation of each parameter's distributions are listed in Table 1.

For each subject, the temporal evolution of the spatially averaged flow through the aortic valve $\left(q_{\text {ave }}(t)\right)$ was taken from the last cardiac cycle, which represents the steady state. The beginning and the end of the ejection are not explicitly regis-

\footnotetext{
${ }^{1}$ Normal distribution is conventionally accepted in medical clinical trials. The $\sigma$ of the normal distribution for each parameter is chosen so that reported normal values are in the $\pm 3 \sigma$ interval.
} 
Table 1: Parameter distributions for virtual populations

\begin{tabular}{cccc}
\hline Population & $\begin{array}{c}\text { Physiological } \\
\text { parameter }\end{array}$ & Unit & $\begin{array}{c}\text { Distribution } \\
\text { parameters }(\mu \pm \sigma)\end{array}$ \\
\hline \hline VP-H \& VP-AS & HR & beats/min & $65 \pm 15 / 3$ \\
VP-H \& VP-AS & SV & mL/beat & $70 \pm 20 / 3$ \\
VP-H \& VP-AS & MAP & $\mathrm{mmHg}$ & $100 \pm 7 / 3$ \\
\hline VP-AS & AVA & $\mathrm{cm}^{2}$ & $1 \pm 1 / 3$ \\
\hline
\end{tabular}

Legend: $\mathrm{VP}-\mathrm{H}=$ healthy virtual population, $\mathrm{VP}-\mathrm{AS}=$ virtual population suffering from aortic stenosis. $\mathrm{HR}=$ heart rate, $\mathrm{SV}=$ stroke volume, $\mathrm{MAP}=$ mean arterial pressure, $\mathrm{AVA}=$ aortic valve area.

tered by the model, so they were identified as time instances when the centerline blood flow through the aortic valve reaches $1 \%$ of the same subject's peak flow. Conversions from flow to velocity and from the average to the maximal value are needed for obtaining realistic values that can be recorded with CW Doppler. The average velocity $v_{\text {ave }}(t)$ of the blood through the orifice can be calculated from the mean flow as:

$$
v_{\text {ave }}(t)=\frac{q_{\text {ave }}(t)}{A}, \quad t \in\left[0, T_{\text {ejec }}\right]
$$

where $A$ denotes the cross-section area of the orifice (ie. the aortic valve area) and $T_{e j e c}$ denotes the end of the ejection interval. Considering the geometry of an open aortic valve, spatial average refers to the arithmetic average of blood flow velocities through an ideal circular orifice, which is in polar coordinates given by:

$$
v_{\text {ave }}(t)=\frac{1}{R^{2} \pi} \int_{\theta=0}^{2 \pi} \int_{r=0}^{R} v(t, r) r d r d \theta
$$

$R$ stands for the radius of the orifice and $r$ is the distance from the center of the orifice to the wall that encloses it.

For a turbulent flow of a fluid through a circular orifice, the fluid dynamics power law equation is applied to approximate the relationship between the jet velocities distributed across the orifice and the maximum velocity (Coulson and Richardson (1999)):

$$
v(t, r)=v_{\max }(t)\left(1-\frac{r}{R}\right)^{\frac{1}{n}}, r \in[0, R]
$$

where $n$ in the exponent is a function of the Reynolds number (Re). The typical Reynolds number for blood flow in the body ranges from 1 in small vessels to 
approximately 4000 in the aorta. For $R e<10^{5}$, the Prandtl's one seventh power law $(n=7)$ is applied to Eq. 3. Plugging the expression for $v(t, r)$ from Eq. 3 with $n=7$ into Eq. 2 leads to the following ratio of the average and the maximal (centerline) velocity:

$$
v_{\max }(t)=\frac{v_{\text {ave }}(t)}{0.817}
$$

After the maximal velocities are extracted, the complete range of velocities lower than and equal to the centerline velocity value - corresponds to the velocity spectrum as captured by CW Doppler. The formed spectral trace is transformed into a binary image and the reduced quality is simulated by adding white noise. All images were then scaled to same dimensions of $100 \times 100$ pixels, as performed with clinical acquisitions.

\subsection{Image registration and atlas construction}

The purpose of an atlas image is to unify a common knowledge about the shape and appearance of an object of interest and - via image registration and segmentation - use it to deduce knowledge for images of similar content. In our case, the object is the aortic outflow profile, obtained from either an echocardiographic scanner or from the computer model. In either case, to construct an atlas, images have to be transformed to a common space. This is achieved via nonrigid registration of prealigned images, as follows.

To set the nomenclature, we will denote an image as $I(t, v)$, where the horizontal axis $t$ of the image corresponds to time and the vertical axis $v$ corresponds to blood flow velocity. If the transform function $T(t, v)=T_{j k}(t, v)$ describes the ideal transformation of pixel coordinates in image $I_{j}(t, v)$ to pixel coordinates in another image $I_{k}(t, v)$, this can be denoted as: $I_{k}(t, v)=I_{j}\left(T_{j k}(t, v)\right)$. Here, $j$ and $k$ denote indices of images in the set.

The objective of the registration process is to find the transform function $T(t, v)$ which maximizes the similarity $S$ between the $j^{t h}$ and the $k^{t h}$ image if coordinates of the $j^{t h}$ image undergo transformation $T(t, v)$ and the $k^{\text {th }}$ image remains intact:

$$
T_{o p t}(t, v)=\arg \max _{T(t, v)} S\left(I_{j}(T(t, v)), I_{k}(t, v)\right)
$$

To simplify the notation, we will further use $S_{j k}$ to stand for $S\left(I_{j}(T(t, v)), I_{k}(t, v)\right)$.

Alignment of ejection periods in all images allows us to parametrize the image domain with $M$ equidistant time points, scaling the $t$-axis to [0,1] via:

$$
t_{m}=\frac{m-1}{M-1}, \forall m=1, \ldots, M
$$


virtually dividing the image into $M-1$ vertical stripes. This allows us to parametrize the transformation vector and initialize it as $f\left(t_{m}\right)=1, \forall m \in[1, M]$. The values between these points are linearly interpolated into $f(t)$, meaning that the transform function is in fact piecewise linear. Alignment of images also means that the overall image deformation function warps the $v$-axis only, effectively applying as velocity scaling: $I(T(t, v))=I(t, f(t) v)$.

Parametrization of the transform function allows us to solve the Eq. 5 by using the gradient ascent optimization algorithm:

$$
f_{i+1}\left(t_{m}\right) \leftarrow f_{i}\left(t_{m}\right)+\eta \nabla S_{j k}\left(f_{i}\left(t_{m}\right)\right)
$$

Here, subscript $i$ stands for the iteration of the optimization process, $\nabla S_{j k}$ describes the increase (or decrease) in similarity of the two images with respect to the change in value of the transformation function parameter and $\eta$ is the convergence rate. The algorithm iteratively updates the transform vector until convergence. The Pearson correlation coefficient is used as an image similarity measure:

$$
S_{j k}=\frac{\operatorname{cov}\left(I_{j}(T(t, v)), I_{k}(t, v)\right)}{\sigma\left(I_{j}(T(t, v))\right) \sigma\left(I_{k}(t, v)\right)},
$$

where $\operatorname{cov}(\cdot)$ is the covariance of two images being compared and $\sigma(\cdot)$ denotes their respective standard deviations. A visualization of the described parametrized non-rigid geometric transformation along the $v$-axis is given in Fig. 3.

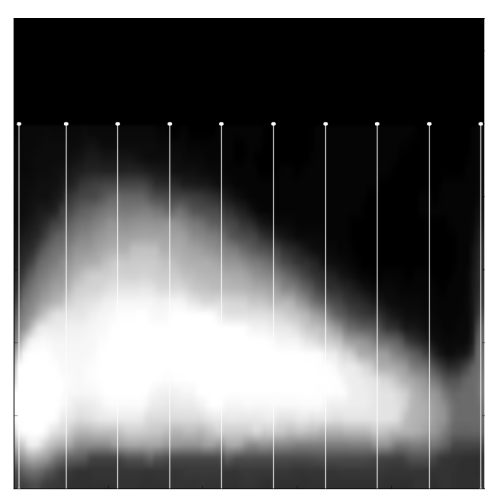

(a)

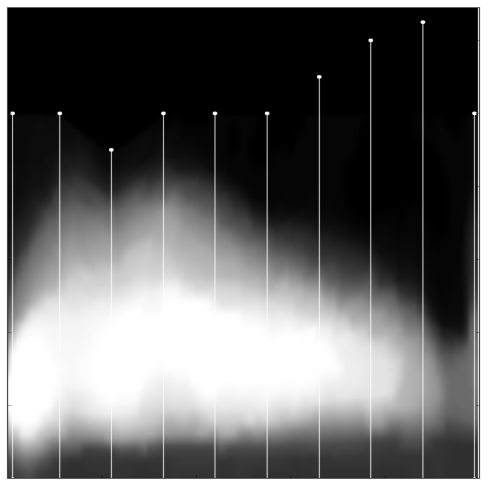

(b)

Figure 3: A visualization of the transformation vector overlaid on an image being registered to a target image: (a) initial values (before deformation), (b) final values (after deformation).

For constructing an atlas, we chose one of the average shape and intensity methods, which aims to preserve a greater amount of details introduced by patient 
variability (Kalinić et al. (2009a)). In short, an image that is least distant from all other images in the training set is assigned to be the root image, i.e. the image to which all the other images are mapped on. Then, an average intensity atlas is created by averaging all images mapped to the root image. With this particular order of operations, the average shape precedes the average intensity.

The distance between images $I_{j}=I_{j}(t, v)$ and $I_{k}=I_{k}(t, v)$ is defined as the total amount of deformation needed for image $I_{j}$ to match image $I_{k}$. We first applied the logarithm to all deformations obtained for the same segment $m$ during the iterative transformation optimization process. The logarithm assures that stretching and squeezing along the $v$-axis by the same factor returns zero deformation. We then applied absolute value to the obtained deformation vector since we do not care if the deformation applies in either one or the other direction. Distance between images $I_{j}$ and $I_{k}$ is then:

$$
d_{j k}=\sum_{m=1}^{M}\left|\sum_{i=1}^{R} \log \left(f_{j k}(t[m], i)\right)\right|
$$

where $M$ is the length of the deformation parameters vector, $R$ is the number of iterations and $f_{j k}(t[m], i)$ denotes the transformation vector in iteration $i$. Distance is calculated for all possible pairs of images in the set.

The image with the least distance from all the others images in the training set is assigned to be the root image $I_{r}$, with $r$ denoting its index in the image set:

$$
r=\arg \min _{k} d_{j k}
$$

Due to the computing limitations (convergence criteria for the optimization process, working with discrete coordinates, interpolation error etc.), transformation of images into the root image is not ideal. It therefore makes sense to average the transformed images into one image that represents all of them simultaneously - the atlas:

$$
A(t, v)=\frac{1}{K} \sum_{j=1}^{K} I_{j}\left(T_{j r}(t, v)\right)
$$

Here, $r$ is the index of the root image, with a segmentation contour selected as the most representative, and $K$ denotes the number of instances in the training set. In the same manner, an average delineation of the atlas is obtained by averaging the delineations of the images from the training set, after they are deformed using the corresponding transformation function. We can conclude that the atlas constructed in this way minimizes bias with respect to the transformation function. 


\subsection{Validation}

\subsubsection{Validation of velocities}

From the aortic outflow velocity, clinicians evaluate the valve condition by calculating the mean and maximal transaortic gradient (Otto (2013)):

$$
\begin{gathered}
\Delta p_{\text {max }} \approx 4 v_{\text {peak }}^{2} \\
\Delta p_{\text {mean }} \approx 4 \frac{\int v^{2} d t}{T_{\text {ejec }}}
\end{gathered}
$$

Unlike the maximal pressure gradient, which is directly proportional to the maximal velocity, the mean pressure gradient takes into account all velocities present during the ejection time $T_{e j e c}$. Since the expected values given with equations (12) and (13) are known for each AS grade (from healthy to severe), they were used for the initial validation of aortic outflow signals obtained with CircAdapt.

\subsubsection{Segmentation validation}

For assessing the segmentation accuracy we used three validation measures that compare the segmentation determined by the proposed method with the ground truth segmentation (manually performed by an expert). Segmentation of the velocity region with respect to the background is a binary problem and allows us to treat the region of interest as a set and use operations and notation from the set theory. To do this, we will denote the velocity region obtained with the proposed atlas-based segmentation with $I_{a}$ and the ground-truth velocity region with $I_{b}$.

The percentage error measures the vertical distance between the contours (ie. the mean absolute distance, MAE) relative with respect to the image height $h$. This corresponds to the size of the non-overlapping area in the segmented images relative with respect to the image size:

$$
e r r=\frac{M A E}{h}=\frac{\left|I_{a} \ominus I_{b}\right|}{h \times w}
$$

Here, $\ominus$ denotes the disjunctive union of the sets, $|\cdot|$ denotes the cardinal number of the set and $w$ denotes the image width.

To avoid the dependence on the image size, we further adopted two measures that consider the segmentation of the region of interest regardless of the size of the background (Taha and Hanbury (2015)). The Dice similarity index (DSI) is a measure of proportion of the overlapping area in two segmented images:

$$
D S I=\frac{\left|I_{a} \bigcap I_{b}\right|}{\left(\left|I_{a}\right|+\left|I_{b}\right|\right) / 2}
$$


For velocity regions of different shapes but similar sizes $\left(\left|I_{a}\right| \approx\left|I_{b}\right|\right)$, the denominator underestimates the non-overlapping region and thus overestimates the similarity. The Jaccard similarity index (JSI), which compares the size of the overlap of the sets with the size of their union, is believed to offer a more reliable measure for evaluating image segmentation:

$$
J S I=\frac{\left|I_{a} \bigcap I_{b}\right|}{\left|I_{a} \cup I_{b}\right|}
$$

Successful segmentation aims for a full overlap of the atlas-based and the manually segmented velocity region $(D S I=1$ and $J S I=1)$, ie. for a zero-displacement between their contours (err $=0$ ).

To determine if there was a statistical significance in error caused by the origin of images used for atlas construction (synthetic vs. classical), we performed twotailed $t$-tests on the corresponding pairs of segmentation scenarios for each valve condition. Tests compared all three error measures, considering $P$-value $>0.05$ an indicator of a statistically significant difference in an error and therefore in the design of the matched scenarios.

\section{Experimental results}

Two virtual populations of patients were created using CircAdapt: 100 healthy individuals and 100 patients with aortic stenosis. The probabilistic distributions of HR, SV, MAP and AVA values characterizing these populations are shown in Figure 4. The velocity contours obtained with CircAdapt for both simulated populations are shown in Figure 5. As expected, the computed Doppler traces belonging to the healthy population are triangular, asymmetrical and early-peaking with lower velocity values, while those belonging to the AS population are more rounded, symmetrical and late-peaking, with higher velocity values proportional to the severity of the disease. The adequacy of the obtained population, according to the expected severity, has been verified with the mentioned diagnostic values. The results are listed in Table 2.

The signals corresponding to aortic outflow blood velocities were then transformed, as described above, into images with CW Doppler properties. 100 images from virtual patients with a healthy aortic valve (VP-H) and 100 images from virtual patients with a stenotic valve (VP-AS) form the two sets of images for the proposed atlas construction approach. The clinical sets consisted of 26 images from healthy volunteers (RP-H) and 114 images from diseased patients (RP-D), respectively. 

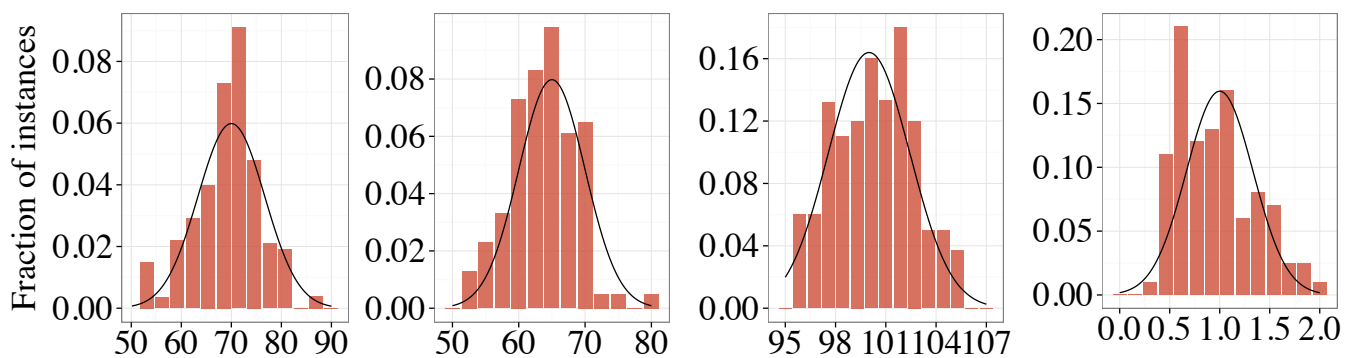

$\mathrm{SV}$ values $[\mathrm{mL} / \mathrm{beat}] \mathrm{HR}$ values [beats/min] MAP values $[\mathrm{mmHg}]$

AVA values $\left[\mathrm{cm}^{2}\right]$

Figure 4: Histograms of obtained parameters values (red bars) fitted with normal distributions defined with parameters in Table 1 (black line).

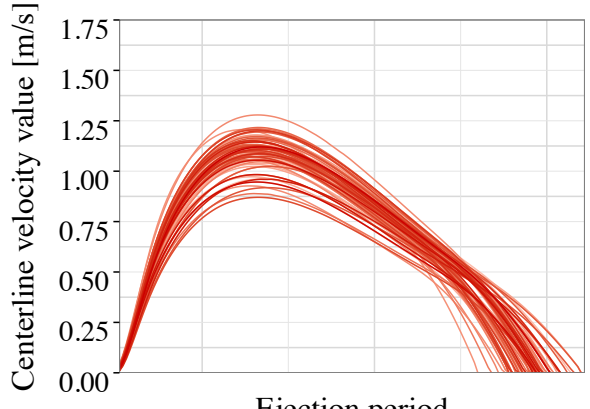

Ejection period

(a)

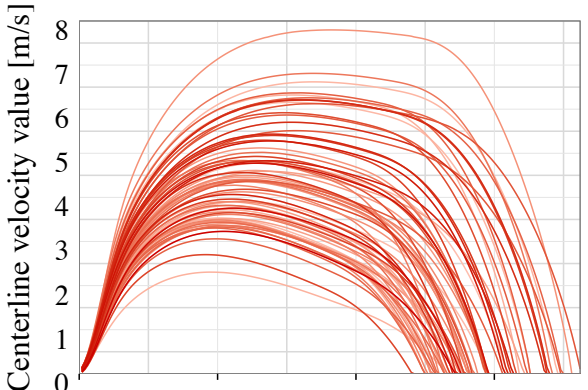

Ejection period

(b)

Figure 5: Set of all centerline velocity contours for each of 100 individuals in the: a) healthy virtual population, b) virtual population with aortic stenosis.

Table 2: Validation of computationally obtained signals

\begin{tabular}{|c|c|c|c|c|c|c|c|c|}
\hline & \multirow{2}{*}{\multicolumn{2}{|c|}{ Healthy }} & \multicolumn{6}{|c|}{ Aortic stenosis } \\
\hline & & & \multicolumn{2}{|c|}{ mild } & $\bmod$ & rate & \multicolumn{2}{|c|}{ severe } \\
\hline Number of patients & \multicolumn{2}{|c|}{100} & \multicolumn{2}{|c|}{7} & \multicolumn{2}{|c|}{35} & \multicolumn{2}{|c|}{58} \\
\hline & $\mathrm{E}$ & $\mathrm{O}$ & $\mathrm{E}$ & $\mathrm{O}$ & $\mathrm{E}$ & $\mathrm{O}$ & $\mathrm{E}$ & $\mathrm{O}$ \\
\hline Area $\left[\mathrm{cm}^{2}\right]$ & $>2$ & 5 & $>1.5$ & 1.728 & $1-1.5$ & 1.224 & $<1$ & 0.717 \\
\hline Jet velocity $[\mathrm{m} / \mathrm{s}]$ & $1-2$ & 1.092 & $2.6-2.9$ & 2.925 & $3-4$ & 3.692 & $>4$ & 5.345 \\
\hline Mean gradient $[\mathrm{mmHg}]$ & NA & 2.482 & $<20$ & 19.941 & $20-50$ & 33.225 & $>50$ & 76.518 \\
\hline Peak gradient $[\mathrm{mmHg}]$ & NA & 4.799 & $<40$ & 34.711 & $40-80$ & 54.930 & $>80$ & 116.812 \\
\hline
\end{tabular}

Legend: $\mathrm{O}=$ obtained mean value, $\mathrm{E}=$ expected value range from Baumgartner et al. (2009). 
For building the atlases, we employed the method that minimizes bias with respect to the transformation function for image-to-atlas registration and applies reduced-resolution strategy for improving the robustness of the registration. Treating each of the image sets as a training set resulted in four atlases shown in Fig. 6. The first two were built using the healthy populations data sets and the last two were created using the data sets of populations with obstructed flow.

Each of the atlases was tested for segmentation of clinical images of healthy volunteers and patients with diagnosed heart condition. Examples of segmentation contours obtained using the proposed atlas against the corresponding ground truth are given in Fig. 7. Percentage error, Dice index and Jaccard index values are given in Table 3 for images of healthy Doppler flow traces and Table 4 for images of obstructed flow traces, describing every possible scenario with regard to combinations of four training and two test sets.

Since we propose simulating condition-specific atlases for segmenting equivalent realistic images, we target scenarios (1) and (7) as the applications of interest that contrast the traditional scenarios (2) and (8), respectively. The results show that when segmenting the images of the normal blood flow, the standard approach of using clinical images of a healthy population for atlas construction (2) gives slightly better segmentations than the atlas based on virtual population (1). Similarly, when segmenting the images of the obstructed flow, the standard approach based on the usage of clinical AS+CAD images for the atlas construction (8) gives slightly better segmentations than the atlas constructed with AS virtual population images (7). Application of atlas created from healthy clinical images for segmenting images that originate from real patients with obstructed aortic flow and vice versa ( 6 and 4 respectively) provide much inferior results. On the contrary, atlases

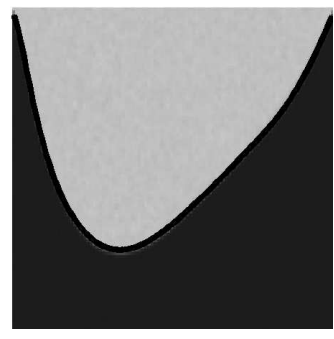

(a)

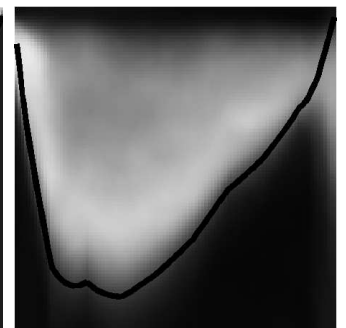

(b)

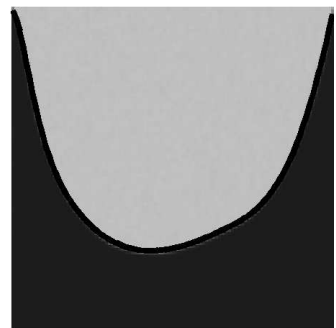

(c)

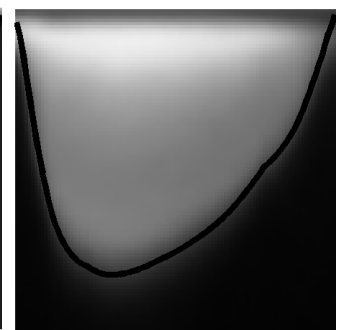

(d)

Figure 6: The atlases built using the datasets of aortic Doppler flow in: a) simulated healthy population, b) real healthy volunteers, c) simulated population with aortic stenosis, d) real population of patients with aortic stenosis and coronary artery disease. All images are in pixels. 


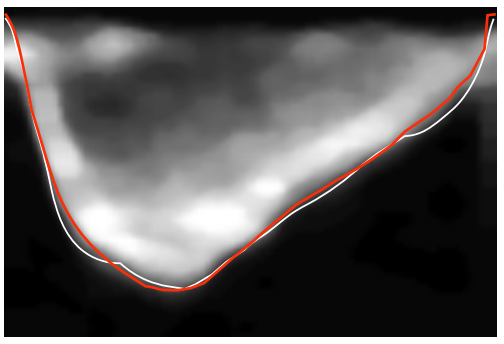

(a)

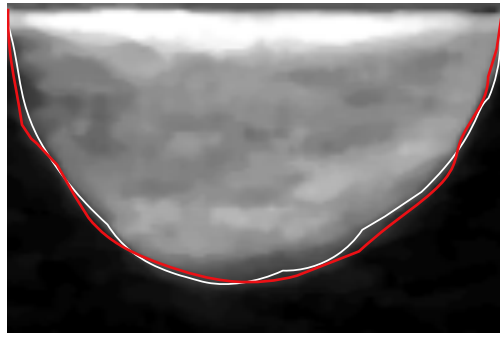

(b)

Figure 7: Examples of Doppler velocity profile images for (a) a healthy valve and (b) a stenotic valve, delineated using the atlas based on VP-AS population (white) vs. its ground truth segmentation (red). Non-zero velocity values at the beginning and the end of the ejection period belong to the background flow (flow present between two ejections intervals, visible in Fig. 2), and are not a part of the ejection outflow.

Table 3: Validation for segmenting Doppler traces belonging to healthy individuals

\begin{tabular}{ccclll}
\hline Scenario & $\begin{array}{c}\text { Training } \\
\text { set }\end{array}$ & $\begin{array}{c}\text { Test } \\
\text { set }\end{array}$ & \multicolumn{1}{c}{ Err (\%) } & \multicolumn{1}{c}{ DSI } & \multicolumn{1}{c}{ JSI } \\
\hline \hline 1 & VP-H & RP-H & $4.989 \pm 1.715^{*}$ & $0.955 \pm 0.023^{*}$ & $0.904 \pm 0.042^{*}$ \\
2 & RP-H & RP-H & $4.315 \pm 1.539$ & $0.965 \pm 0.015$ & $0.933 \pm 0.062$ \\
3 & VP-AS & RP-H & $3.942 \pm 1.817^{* *}$ & $0.965 \pm 0.022^{* *}$ & $0.933 \pm 0.061^{* *}$ \\
4 & RP-D & RP-H & $17.445 \pm 5.220$ & $0.819 \pm 0.034$ & $0.461 \pm 0.275$ \\
\hline
\end{tabular}

Legend: $\mathrm{VP}-\mathrm{H}=$ healthy virtual population, VP-AS = virtual population suffering from aortic stenosis, RP$\mathrm{H}=$ real healthy population, $\mathrm{RP}-\mathrm{D}=$ real population of cardiac patients, $\mathrm{Err}=$ percentage error, DSI $=$ Dice similarity index, JSI = Jaccard similarity index. Results are presented as mean value \pm standard deviation. Superscripts * and ${ }^{* *}$ denote experiments with $P$-value $>0.05$ and $<0.001$, respectively, for each proposed VP scenario as compared to its corresponding reference RP scenario.

Table 4: Validation for segmenting Doppler traces belonging to patients

\begin{tabular}{ccclll}
\hline Scenario & $\begin{array}{c}\text { Training } \\
\text { set }\end{array}$ & $\begin{array}{c}\text { Test } \\
\text { set }\end{array}$ & \multicolumn{1}{c}{ Err (\%) } & \multicolumn{1}{c}{ DSI } & JSI \\
\hline \hline 5 & VP-H & RP-D & $5.642 \pm 0.238^{* *}$ & $0.948 \pm 0.018^{* *}$ & $0.898 \pm 0.188^{* *}$ \\
6 & RP-H & RP-D & $16.23 \pm 3.166$ & $0.905 \pm 0.013$ & $0.786 \pm 0.213$ \\
7 & VP-AS & RP-D & $4.327 \pm 1.219^{*}$ & $0.963 \pm 0.015^{*}$ & $0.929 \pm 0.019^{*}$ \\
8 & RP-D & RP-D & $4.256 \pm 1.988$ & $0.964 \pm 0.011$ & $0.923 \pm 0.021$ \\
\hline
\end{tabular}


based on simulated datasets (1, 3, 5 and 7), even in cases which theoretically have the largest difference between the training and the test sets ( 3 and 5), always provide a reasonable performance. The reasoning behind these results is discussed in the following section.

\section{Discussion}

In this paper, we propose a more efficient approach for atlas construction by introducing virtual patient data sets - simulated with a computational model of the circulation - to support atlas-based segmentation of (Doppler) medical images. The method was validated on two sets of collected clinical images and compared with the standard methods of atlas construction, resulting in an overall good performance of our proposed approach.

For segmenting images that represent a population with a same valve condition as the atlas (scenarios 1 vs. 2 for healthy and 7 vs. 8 for obstructed flow), we obtained a slightly better result when we used an atlas constructed with clinical images. This was expected since the classical approach in this case features the overlap of the data used for atlas construction and the segmentation, whereas the segmentation of the proposed approach is completely unbiased to testing data. However, the difference between the mean segmentation errors obtained with the classical and the new approach has shown to be practically negligible. T-tests for all error measures yielded $P$-values larger than the significance level (0.05), thus accepting the hypothesis that there is no significant difference in the atlas performance caused by the image origin for the described scenarios. This confirms our initial idea for constructing disease-specific atlases with simulated outflow profile images, which greatly simplifies the process as compared to a clinical setting.

When the clinical images used to create an atlas do not represent the same disease process as the images that are being segmented, the atlases based on simulated images in fact outperformed the atlases based on the clinical images (scenarios 3 vs. 4 and 5 vs. 6). The apparent difference in error for these scenarios is complemented with low $P$-values $(<0.001)$, favouring the proposed approach for cross-content image segmentation. Moreover, the overall underperformance of the atlas created using a classical approach in the scenario 4 is a result of combining an insufficiently large training data set with the cross-content image segmentation. Since smaller sets can capture only a limited amount of realistic variability, this demonstrates the importance of the size of the data set used to create an atlas.

Comparison with our previous work (Baličević et al. (2013)), where we tested the concept on a healthy population, shows that the average intensity atlas out- 
performs in segmentation when the images in the training set do not differ significantly. This corresponds to the atlas created with images of healthy virtual individuals (scenario 1). Since the segmentation contours show smaller variability in maximal velocity values and shapes, as can be seen in Fig.5, we thus obtained better results ( $4.18 \%$ for Err and 0.958 for DSI). In cases with greater variability between images, the same approach would result in an atlas with less details and a more fuzzy boundary (Kalinić et al. (2009a)), leading us to the presented more complex approach for atlas construction. This explains the superior performance of the atlas created from virtual patients suffering from aortic stenosis (scenario 3 and 7).

Finally, we can conclude that the presented atlases formed from computationally simulated datasets resulted in highly accurate segmentations of all types of clinical aortic outflow images. However, for obtaining the best possible segmentation of images corresponding to different severities of the disease, it is better to use not only the same type of images, but also an atlas construction method appropriate for the image content. Statistical tests showed that the origin of these images (whether it is clinical or simulated) does not change the accuracy of the segmentation significantly, suggesting that the concept proposed for segmenting aortic valve velocity profile images can serve as a qualified alternative to stateof-the-art approaches. As such, it can be used to pursue automatizing of profile segmentation for more complex Doppler shapes, ie. for mitral and tricuspid valves or pulmonary veins.

\section{Conclusions}

In this paper, we presented a novel approach for constructing an anatomical (both shape and appearance) atlas based on computationally simulated images for segmenting aortic valve Doppler images. The general idea consists of integrating an existing atlas construction methods with a computer model capable of realistic simulations of the human circulation.

Apart from apparent contributions such as consistency and gain in speed over the manual approach, there are several reasons for introducing the computational simulations in the process. Since the clinical imaging, as well as the following research, is related to a specific condition, collecting the images is dependent on the number of available patients. Next, for creating the anatomical atlas, the diversity of the same condition within the patient population is relevant. With a small number of patients, the chance of covering all possible cases is reduced, resulting in a non-representative data set with characteristics that do not follow 
an expected normal distribution. Introducing the computational model allows for producing larger image data sets, with a statistically grounded control over the parameter distributions in the set.

In order to capture the expected variability for a certain disease, it is crucial that the computational model can simulate the given disease accurately. The model that we used for generating the artificial profiles is CircAdapt, previously validated for simulating the hemodynamics and vascular structures mechanics for different heart conditions. Despite its great potential and capabilities, the clinical translation from CircAdapt is not straightforward given that it results in a selected set of one-dimensional signals, while the common clinical acquisition of flow information results in 2D images. Fluid dynamics of the blood flow combined with image processing methods allows us to link the model to the atlas construction method and form a hybrid framework for a fast, accurate, consistent and reproducible segmentation of clinically acquired aortic blood outflow images.

While this paper is a proof of concept for what indeed is a relatively simple, yet common and significant task in clinical practice, the possibilities of the proposed concept are numerous. With the described comprehensive explanation, we set the grounds for extending the application towards the automated segmentation of other Doppler signals where getting manual segmentation has proven to be a problematic and demanding task and would benefit from the presented approach.

\section{Acknowledgement}

Research leading to these results has received funding from the Ministry of Science, Education and Sports, Republic of Croatia (036-0362214-1989), Subprograma de Proyectos de Investigacin en Salud (FIS), Instituto de Salud Carlos III, Spain (ref. PI11/01709); the Spanish Ministry of Economy and Competitiveness (grant TIN2014-52923-R) and FEDER and the Seventh Framework Programme (FP7/2007-2013) under grant agreement No. 611823. Additionally, we would like to thank Frank Weidemann for a subset of the patient images, and Catalina Tobon-Gomez and Georgina Palau-Caballero for provided CircAdapt support.

\section{References}

Arts, T., Delhas, T., Bovendeerd, P., Verbeek, X., Prinzen, F., 2005. Adaptation to mechanical load determines shape and properties of heart and circulation: the Circ-Adapt model. In: Am J Physiol Heart Circ Physiol 288(4): 1943-1954. 
Baličević, V., Kalinić, H., Lončarić, S., Čikeš, M., Palau-Caballero, G., TobonGomez, C., Bijnens, B.H., 2013. Atlas construction for cardiac velocity profiles segmentation using a lumped computational model of circulatory system. In: Proc FIMH 2013.

Baumgartner, H., Hung, J., Bermejo, J., Chambers, J.B., Evangelista, A., Griffin, B.P., Iung, B., Otto, C.M., Pellikka, P.A., Quiones, M., 2009. Echocardiographic assessment of valve stenosis: EAE/ASE recommendations for clinical practice. In: Eur J Echocardiogr, 10(1), 1-25.

Bermejo, J., Antoranz, J.C., García-Fernández, M.A., Moreno, M.M., Delcán, J.L., 2000. Flow dynamics of stenotic aortic valves assessed by signal processing of doppler spectrograms. In: Am J Cardiol 2000, 611-617.

Cavalcanti, S., Cavani, S., Ciandrini, A., Avanzolini G., 2004. Mathematical modeling of arterial pressure response to hemodialysis-induced hypovolemia In: Computers in Biology and Medicine, Vol. 36(2), 128144.

Čikeš, M., Kalinić, H., Baltabaeva, A., Lončarić, S., Parsai, C., Miličić, D., Čikeš, I., Sutherland, G., Bijnens, B., 2009. The shape of the aortic outflow velocity profile revisited: is there a relation between its asymmetry and ventricular function in coronary artery disease? In: Eur J Echocardiogr 10(7), 847-57.

Coulson, J.M., Richardson, J.F., 1999. Chemical engineering: Fluid flow, heat transfer and mass transfer, 83-85. Elsevier Science \& Technology Books, Oxford.

De Lazzari, C., Darowski, M., Ferrari, G., Clemente, F., Guaragno, M., 2000. Computer simulation of haemodynamic parameters changes with left ventricle assist device and mechanical ventilation. In: Computers in Biology and Medicine, Vol. 30(2): 5569.

Di Molfetta, A., Santini, L., Forleo, G.B., Cesario, M., Tota, C., Sgueglia, M., Sergi, D., Ferrari, G., Romeo, F., 2010. Use of a comprehensive numerical model to improve biventricular pacemaker temporization in patients affected by heart failure undergoing to CRT-D therapy. In: Med Biol Eng Comput 48(8): 755-64.

Gaillard, E., Kadem, L., Clavel, M., Pibarot, P., Durand, L., 2010. Optimization of Doppler Echocardiographic Velocity Measurements Using an Automatic Contour Detection Method Ultrasound in Medicine \& Biology, 36(9), 1513-1524. 
Greenspan, H., Shechner, O., Scheinowitz, M., Feinberg, M., 2005. Doppler echocardiography flow-velocity image analysis for patients with atrial fibrillation, Ultrasound Med Biol. 31, 1031-1040.

Gruslys, A., Sawiak, S., Ansorge, R, 2011. 3000 non-rigid medical image registrations overnight on a single PC. In: Nuclear Science Symposium and Medical Imaging Conference 2011 (NSS/MIC), 3073-3080.

Gubern-Mérida, A., Kallenberg, M., Mart, R., Karssemeijer, N., 2011. Multiclass Probabilistic Atlas-Based Segmentation Method in Breast MRI. In: Proc of IbPRIA 6669: 660-667.

Headley, Jan M., 2002. Invasive Hemodynamic Monitoring: Physiological Principles and Clinical Applications. Edwards Lifesciences.

Kalinić, H., 2008. Atlas-based image segmentation: A Survey. Department of Electronic Systems and Information Processing, Universiy of Zagreb.

Kalinić, H., Lončarić, S., Čikeš, M., Miličić, D., Bijnens, B., 2009. Comparison of Different Methods for Atlas Construction In: 17th European Signal Processing Conference (EUSIPCO): 1319-1323.

Kalinić, H., Lončarić, S., Čikeš, M., Miličić, D., Čikeš I., Sutherland, G., Bijnens, B., 2009. A method for registration and model-based segmentation of Doppler ultrasound images. In: Proceedings of SPIE Medical Imaging, 7259-27.

Kalinić, H., Lončarić, S., Čikeš, M., Miličić, D., Bijnens, B., 2012. Image registration and atlas-based segmentation of cardiac outflow velocity profiles. In: Comput Methods Programs Biomed, 106(3), 188-200.

Khalafvand, S.S., Ng E.Y.K., Zhong, L., Hung, T.K., 2012. Fluid-dynamics modelling of the human left ventricle with dynamic mesh for normal and myocardial infarction: Preliminary study. In: Computers in Biology and Medicine, Vol. 42(8), 863870.

Kuijpers, N.H.L., Dassen, W., van Dam, P.M., van Dam, E.M., Hermeling, E., Lumens, J., Arts, T., Delhaas, T., 2012. CircAdapt: a User-Friendly Learning Environment for (Patho) physiology of Heart and Circulation. In: Computing in Cardiology (CinC), 39, 969-972. 
Kuijpers, N.H., Hermeling, E., Lumens, J. et al., 2014. Mechano-electrical coupling as framework for understanding functional remodeling during LBBB and CRT. In: Am J Physiol Heart Circ Physiol 306, H1644-H1659.

Kuklisova-Murgasova, M., Aljabar, P., Srinivasan, L., Counsell, S.J., Doria, V., Seraga, A., Gousias, I.S., Boardman, J.P., Rutherfordb, M.A., Edwards, A.D., Hajnal, J.V., Rueckert, D., 2011. A dynamic 4D probabilistic atlas of the developing brain. NeuroImage 54 (4), 27502763.

Kupfahl, C., Honold, M., Meinhardt, G., Vogelsberg, G., Wagner, A., Mahrholdt, H., Sechtem, U., 2004. Evaluation of aortic stenosis by cardiovascular magnetic resonance imaging: Comparison with established routine clinical techniques. Heart 90, 893-901.

La Gerche, A., MacIsaac, A.I., Burns, A.T., Mooney, D.J., Inder, W.J., Voigt, J.U., Heidbchel, H., Prior, D.L., 2010. Pulmonary transit of agitated contrast is associated with enhanced pulmonary vascular reserve and right ventricular function during exercise. In: J Appl Physiol 109(5): 1307-17.

Leenders, G.E., Lumens, J., Cramer, M.J., De Boeck, B.W., Doevendans, P.A., Delhaas, T., Prinzen, F.W., 2012. Septal deformation patterns delineate mechanical dyssynchrony and regional differences in contractility: analysis of patient data using a computer model. In: Circ Heart Fail 5: 87-96.

Liang, F., Takagi, S., Himeno, R., Liu, H., 2009. Multi-scale modeling of the human cardiovascular system with applications to aortic valvular and arterial stenoses. In: Med Biol Eng Comput 47(7): 743-755.

Lumens, J., Delhaas, T., Kirn, B., Arts, T., 2008. Modeling ventricular interaction: a multiscale approach from sarcomere mechanics to cardiovascular system hemodynamics. In: Pac Symp Biocomput, 378-89.

Lumens, J., Delhaas, T., Kirn, B., Arts, T., 2009. Three-wall segment (TriSeg) model describing mechanics and hemodynamics of ventricular interaction. In: Ann Biomed Eng 37(11), 2234-2255.

Lumens, J., Delhaas, T., 2012. Cardiovascular modeling in pulmonary arterial hypertension: focus on mechanisms and treatment of right heart failure using the CircAdapt model. In: Am J Cardiol 110, 39S-48S. 
Lumens, J., Blanchard, D.G., Arts, T., Mahmud, E., Delhaas, T., 2010. Left ventricular underfilling and not septal bulging dominates abnormal left ventricular filling hemodynamics in chronic thromboembolic pulmonary hypertension. In: Am J Physiol Heart Circ Physiol, 299(4), H1083-91.

Ma, Z., Tavares, J.M., Jorge, R.N., Mascarenhas, T., 2010. A Review of Algorithms for Medical Image Segmentation and their Applications to the Female Pelvic Cavity. In: Comput Methods Biomech Biomed Engin, 13(2), 235-46.

Madden, M.J., 2007. Segmentation of images with low-contrast edges (Thesis No. 1451721). M.S.E.E., West Virginia University, 115 pages.

Margulescu, A.-D., Cinteza, M., and Vinereanu, D., 2006. Reproducibility in echocardiography: Clinical significance, assessment, and comparison with other imaging methods. Maedica, 1, 29-34.

MATLAB and Statistics Toolbox Release 2010b, The MathWorks, Inc., Natick, Massachusetts, United States.

McCormick, M., Nordsletten, D., Lamata, P., Smith, N.P., 2014. Computational analysis of the importance of flow synchrony for cardiac ventricular assist devices. In: Computers in Biololgy and Medicine 49, 83-94.

NSR Physiome Project, NSR Physiome Models. Last modified: 2012. http://www.physiome.org/Models. Accessed: June 2012.

Otto, C., 2013. Textbook of Clinical Echocardiography. (5th ed.). Philadelphia, PA: Elsevier/Saunders.

Palau-Caballero, G., Tobon-Gomez, C., Baličević, V., Butakoff, C., Lončarić, S., Sitges, M., Bijnens, B.H., 2013. Improving clinical translation of cardiovascular circulatory models through an intuitive Graphical User Interface to CircAdapt, presenting simulation results as clinical images and signal. In: Proc STACOM Workshop at MICCAI 2012, Springer

Pham, D.L., Xu, C.,Prince, J.L., 2000. Current methods in medical image segmentation. In: Annu Rev Biomed Eng, 2, 315-37.

Shi, Y., Lawford, P., Hose, R., 2011. Review of Zero-D and 1-D Models of Blood Flow in the Cardiovascular System. In: BioMedical Engineering OnLine, 10(33). 
Taha, A.A., Hanbury, A., 2015. Metrics for evaluating 3D medical image segmentation: analysis, selection, and tool In: BMC Med. Imaging 15(1), 29.

Tobon-Gomez, C., Sukno, F.M., Butakoff, C., Huguet, M., Frangi, A.F., 2012. Automatic training and reliability estimation for 3D ASM applied to cardiac MRI segmentation. In: Phys Med Biol 57(13), 4155-74.

Tobon-Gomez, C., Butakoff, C., Aguade, S., Sukno, F., Moragas, G., Frangi, A.F., 2008. Automatic construction of 3D-ASM intensity models by simulating image acquisition: application to myocardial gated SPECT studies. In: IEEE Trans Med Imaging 27(11), 1655-67.

Tschirren, J., Lauer, R.M., Sonka, M., 2001. Automated analysis of doppler ultrasound velocity flow diagrams. In: IEEE Trans. Med. Imaging 2001, 20(12), 1422-1425.

Yishan, L., Chung, A.C.S., 2011. An atlas-based deep brain structure segmentation method: from coarse positioning to fine shaping. In: Proc IEEE Int Conf Acoust Speech Signal Process (ICASSP), 1085-1088.

Wang, H., Suh, J.W., Das, S.R., Pluta, J.B., Craige, C., Yushkevich, P.A., 2013. Multi-Atlas Segmentation with Joint Label Fusion. IEEE Trans Pattern Anal Mach Intell 35(3), 611-623. 